\title{
Numerical simulations of highly collimated protostellar outflows
}

\section{The effects of relative density}

\begin{abstract}
A. Rosen and M. D. Smith
Armagh Observatory, College Hill, Armagh BT61 9DG, Northern Ireland, UK

e-mail:mds@star.arm.ac.uk

Received 14 February 2003 / Accepted 1 October 2003

Abstract. We present three-dimensional hydrodynamic simulations of jets as a model for protostellar outflows. We investigate molecular jets which are initially heavier, equal or lighter than a uniform ambient molecular medium, as well as a ballistic atomic jet, with the aim of distinguishing the resulting structures and relating them to various proposed protostellar evolutionary stages. We modify the ZEUS numerical code, to include time-dependent molecular hydrogen chemistry, a limited equilibrium $\mathrm{C}$ and $\mathrm{O}$ chemistry, and a detailed cooling function. We find highly focussed and accelerated flow patterns for outflows driven by molecular jets, caused by the combined strong cooling, small imposed jet shear and precession. We also find shoulders in the interface with associated shocks visible in our simulated near-infrared $\mathrm{H}_{2}$ images. The shoulder location relative to the front of the bow shock distinguishes the relative density. Apart from this, the outflow structures are quite similar provided the jet is molecular. The ratio of jet power to $\mathrm{H}_{2} 1-0 \mathrm{~S}(1)$ line luminosity (increasingly required to interpret observations), is generally in the range 80-600. Sub-millimetre CO properties, including a velocity-position and velocity-channel diagram; are presented. We compare mass-velocity relationships derived directly and via the simulated $\mathrm{CO}$ data: significant systematic differences are uncovered. For the future, we identify fine-scale structure in the rotational CO 2-1 and CO 14-13 rotational lines which can be resolved with the millimetre array ALMA and the Herschel (FIRST) Observatory. We identify highly collimated outflows in the near-infrared that can be interpreted by this model.
\end{abstract}

Key words. hydrodynamics - shock waves - ISM: clouds - ISM: molecules

\section{Introduction}

Powerful supersonic outflows occur during the formation of a star (Bally \& Lada 1983; Bachiller 1996; Richer et al. 2000). The outflows may possess highly collimated components, in the form of twin jets. The jets are observed as a trail of high speed emission knots, consisting of shock-compressed atomic and molecular gas (Reipurth et al. 2000; Reipurth \& Bally 2001). The jets propagate through bipolar lobes, which may partly consist of the accumulated gas that has been expelled through the jets, partly swept-up gas and partly deposited from a prototstellar wind (Lee et al. 2000, 2002). Prominent examples of jet-driven collimated outflows include HH 111 (Nagar et al. 1997; Hartigan et al. 2001), HH 211 (Gueth \& Guilloteau 1999; Chandler \& Richer 2001), HH 212 (Lee et al. 2000) and HH 288 (Gueth et al. 2001). For many other less resolved outflows, there is evidence that their momentum also derives from the thrust of jets although wider winds may well contribute (Ray 2000).

Prominent outflows with ordered structure may promote our understanding of the properties of the driving sources. Often, the discovery of an outflow precedes the discovery of

Send offprint requests to: A. Rosen, e-mail: rar@star.arm. ac.uk a newly forming star because the deeply embedded protostar is obscured (e.g. Stanke et al. 2002). Moreover, the outflows are most powerful while a protostar grows in mass through accretion from the obscuring environment (Bontemps et al. 1996; Saraceno et al. 1996; Nisini et al. 2002b). We thus suspect that outflows evolve on similar time scales as those relevant to their protostars. In particular, mass inflow and mass outflow rates are closely related to protostellar Class, with the highest mass flow rates being achieved during the Class 0 birth stage.

We have undertaken a sequence of hydrodynamic simulations, motivated by the need for predictions from the general scenario of an evolving jet-driven outflow. Here, we present a sequence involving the jet density relative to the ambient molecular cloud density. We wish to test the hypothesis that the transition from overdense to underdense jets follows the Class 0, Class 1 and Class 2 transitions of the protostar. More precisely, however, the runs predict the structure produced from non-evolving molecular jets from protostars that will proceed to form stars with a range of masses, while embedded within environments of different depth. Simulations of truly evolving jets, during which we gradually increase or decrease the outflowing jet density, have been presented in another paper (Rosen \& Smith 2003). 
Many previous numerical studies of jets from young stars have examined the relatively weak later stages. Several simulations have treated atomic gas with low mass outflow rates and so required less extreme physical modelling. One jet of material is injected, to form one lobe in half the cloud, rather than the observed twin-jet bipolar outflow structures. The simulations have demonstrated that collimated jets maintain their collimation, with stronger radiative cooling tending to further focus the flow pattern (Blondin et al. 1990; de Gouveia dal Pino \& Benz 1993). The jet interacts with the environment only at the impact location where a prominent bow shock propagates. The ambient material is pushed forward and transversely away by the bow to form a lobe. The jet boundary is thus protected from the lobe by a non-interacting cocoon of warm but low pressure gas. The jet is only observable in optical emission lines when supersonic pulsations are superimposed (Raga et al. 1991; Stone \& Norman 1993b). Numerical studies of radiatively cooled jets are now progressing by involving initial magnetic field configurations (Frank et al. 1998; Cerqueira \& de Gouveia dal Pino 1999, 2001; Gardiner et al. 2000; O'Sullivan \& Ray 2000) and input conditions consistent with the rotation and shear of accretion disk processes (Frank et al. 2000).

In order to simulate powerful molecular outflows, molecular hydrogen dissociation and molecular cooling have been added to numerical codes (Raga et al. 1995; Downes \& Ray 1999; O’Sullivan \& Ray 2000). The discovery of many highthrust molecular jets led to detailed simulations in which a restricted hydrogen chemistry, including thermal dissociation, molecule formation on dust, ro-vibrational cooling, $\mathrm{CO}$ rotational and gas-grain cooling were introduced (Suttner et al. 1997a). Three dimensional simulations of ballistic jets with small opening and precession angles generated structures resembling many outflows in the infrared and submillimetre, but too highly collimated to be consistent with well-known outflows such as the NGC 2264 outflow (Smith et al. 1997). Nevertheless, outflows from both low mass (e.g. IRAS 05155+0707, Lee et al. 2002) and high-mass protoststellar objects (e.g. IRAS $05358+3543$, Beuther et al. 2002) do show high collimation.

Further simulations of ballistic jets, involving highamplitude (hammer) pulsations, high shear and spray, predicted a variety of wider structures, proper motions and spectroscopic results (Völker et al. 1999). In these $100 \mathrm{~km} \mathrm{~s}^{-1}$ jet simulations, molecules are dissociated at the tips of the bow shocks. Molecules are also destroyed in the jet as they enter freshlyformed shock waves as pulsations steepen. Molecules survive the shocks further downstream since the strength of the pulses decay rapidly (Suttner et al. 1997a).

These simulations are complementary to the axisymmetric molecular jet simulations of higher resolution but lower jet densities of Downes \& Ray (1999) and Downes \& Cabrit (2003), and the one-dimensional simulations with a complex molecular chemistry of Williams et al. (e.g., Lim et al. 2002). The lower jet densities used in the approach of Downes et al. contrast with those determined in molecular protostellar jets. Molecular densities are derived from analyses of near-infrared excitation and extinction, as well as being inferred from flux levels. Derived molecular densities range from $10^{4} \mathrm{~cm}^{-3}$ in outflows from lowmass protostars like HH 111 to above $10^{6} \mathrm{~cm}^{-3}$ in the OMC-1 outflow (Nisini et al. 2002a; Vannier et al. 2001). The density is critical in determining the cooling length behind the shock and this, subsequently, controls the nature of the flow (Blondin et al. 1990).

Studies of outflows generally conclude that C-type shocks are responsible for the emission and acceleration properties of excited molecular hydrogen. For this reason, our $\mathrm{H}_{2}$ analysis presented here, based on J-type radiative shocks, must be considered with caution. The higher resolution obtained when employing a lower density may help to mimic the width of a C-type shock in which the shock is indeed broadened by the interaction with ion-magnetosonic waves. In this case, however, the shock surface is subject to C-shock instabilities and, due to the different acceleration mechanism, molecular hydrogen velocities are not predictable.

In addition Downes \& Cabrit (2003) compute the massvelocity and molecular line-intensity relationships, but the line emission is calculated applying methods that are better suited for higher density regions (for the $\mathrm{CO}$ emission, this is stated in Downes \& Ray 1999). It is also not clear how the $\mathrm{H}_{2}$ can be significantly vibrationally excited at such low densities. Finally, we also note that $2 \mathrm{D}$ and $3 \mathrm{D}$ simulations generate different outflow morphologies with axisymmetric simulations generating wider outflows (Suttner et al. 1997a).

Accumulating observations, indicating a wide range of outflow properties, raise new concerns. The following are examples. (1) Not all jets are ballistic. The HH 34 jet could become lighter than ambient gas, leading to a deceleration with distance (Devine et al. 1997). Spectra of GGD 34 indicate that the jet is lighter than the ambient medium (Gómez de Castro $\&$ Robles 1999). How can we characterise the relative density? (2) Gueth et al. (2001) remark upon the striking resemblance between numerically-derived images and the HH 288 outflow but call for detailed $\mathrm{CO}$ velocity channel maps to facilitate a full comparison. (3) The bipolar outflows often contain massive reservoirs of relatively slow-moving gas. In terms of derived mass-velocity distributions of the form $m \propto v^{-\gamma}$, values of $\gamma$ larger than 2 are not uncommon (e.g. Ridge \& Moore 2001). Such steep distributions were not found in the above simulations but it was suggested that they would occur if dynamically older outflows could be simulated. (4) Line profiles of molecular hydrogen emission can now also be quantitatively analysed. Recently, Salas \& Cruz-González (2002) have examined the velocity distribution integrated over the entire source. $\mathrm{H}_{2}$ line profiles were indeed presented from simulations by Suttner et al. (1997a), albeit over different parts of the flow, and spatial variations were predicted. The new data allow a quantitative approach. (5) Wide-field near infrared cameras reveal long chains of $\mathrm{H}_{2}$ knots with extremely high collimation (e.g., HH 34, Stanke et al. 2002 and within CB 34, Khanzadyan et al. 2002). (6) Molecular hydrogen emission from shocks in Class 0 protostellar outflows is on average ten times stronger than that from Class 1 outflows (Stanke 2000). Under what conditions can molecular jets radiate efficiently in the $\mathrm{H}_{2}$ emission lines?

We intend here to improve on the molecular physics contained in fully $3 \mathrm{D}$ jet simulations to date, to cover a wider 
Table 1. Jet and ambient medium parameters.

\begin{tabular}{lccrccccc}
\hline \hline Run & $\eta^{a}$ & $\begin{array}{c}\rho_{\mathrm{j}} \\
\mathrm{g} \mathrm{cm}^{-3}\end{array}$ & $\kappa^{b}$ & $\begin{array}{c}T_{\mathrm{j}} \\
\mathrm{K}\end{array}$ & $M_{\mathrm{j}}$ & $\begin{array}{c}\text { Mass flux } \\
M_{\odot} \mathrm{yr}^{-1}\end{array}$ & $\begin{array}{c}\text { Thrust }^{c} \\
\mathrm{~km} \mathrm{~s}^{-1} M_{\odot} \mathrm{yr}^{-1}\end{array}$ & $\begin{array}{c}\text { Power }^{c} \\
L_{\odot}\end{array}$ \\
\hline Light & 0.1 & $2.32(-20)$ & 1 & 100 & 140 & $3.13(-8)$ & $3.05(-6)$ & 0.025 \\
Equal & 1.0 & $2.32(-20)$ & 10 & 100 & 140 & $3.13(-8)$ & $3.05(-6)$ & 0.025 \\
Dense & 10.0 & $2.32(-19)$ & 100 & 100 & 140 & $3.13(-7)$ & $3.05(-5)$ & 0.25 \\
Atomic & 10.0 & $2.32(-19)$ & 100 & 100 & 105 & $3.13(-7)$ & $3.05(-5)$ & 0.25 \\
\hline
\end{tabular}

${ }^{a}$ Ratio of jet-to-ambient density.

${ }^{b}$ Ratio of jet-to-ambient pressure (or internal energy density, assuming a constant adiabatic index).

${ }^{c}$ The velocity moments of the jet are time averages over one cycle of the velocity pulsation and do include the radial shear of the axial velocity.

range of jet injection conditions and to improve the computational resolution and grid size (see Sect. 2). We present nonballistic jet conditions, while maintaining high densities relevant to molecular gas. We also present the case of a largely atomic jet propagating into a molecular medium. We also increase the resolution, add a detailed cooling function, and introduce equilibrium $\mathrm{C}$ and $\mathrm{O}$ chemistry as a first approximation. Magnetic field and radiative transfer properties are neglected. Also, we restict the discussion here to involve uniform environment, non-evolving pulsed jet and narrow precession angles.

\section{ZEUS-3D with molecular cooling and chemistry}

We have modified the explicit ZEUS-3D code to include a semi-implicit method for calculating the molecular and atomic hydrogen fractions. We have now added a limited equilibrium $\mathrm{C}$ and $\mathrm{O}$ chemistry to calculate the $\mathrm{CO}, \mathrm{OH}$ and $\mathrm{H}_{2} \mathrm{O}$ abundances. Equilibrium $\mathrm{CO}$ and $\mathrm{H}_{2} \mathrm{O}$ is a reasonable approximation in non-dissociative shocks where the $\mathrm{H}_{2}$ content also ensures $\mathrm{CO}$ and $\mathrm{H}_{2} \mathrm{O}$ are fully formed. It is also appropriate in the cloud gas where these molecules have time to reach equilibrium. In dissociative shocks, however, the cooling and equilibrium time scales are comparable and, at our chosen densities, non-equilibrium chemistry is strictly required as the gas cools from $8000 \mathrm{~K}$ to $1000 \mathrm{~K}$, in particular for densities lower than $10^{6} \mathrm{~cm}^{-3}$ (Smith \& Rosen 2003). On the positive side, these shocks do not significantly contribute to the molecular emission. On the negative side, equilibrium chemistry leads to a wider range of conditions under which shocks are unstable. Note here that UV radiation, magnetic fields and molecule depletion onto grains will also all play major roles in the cooling layers of dissociative shocks but are ignored here.

The cooling and heating rates are presented in Smith \& Rosen (2003). We include cooling through rotational and vibrational transitions of $\mathrm{H}_{2}, \mathrm{CO}$, and $\mathrm{H}_{2} \mathrm{O}, \mathrm{H}_{2}$ dissociative cooling and reformation heating, gas-grain cooling/heating, and a time-independent atomic cooling function that includes nonequilibrium effects. We assume that the gas includes an additional $10 \%$ by number of helium atoms, so the mean atomic mass is $2.32 \times 10^{-24} \mathrm{~g}$. The dust temperature is fixed at $20 \mathrm{~K}$.

We analyse the results from four three-dimensional simulations, which include a sequence of three molecular simulations that differ in jet-to-ambient density ratio, $\eta \equiv \rho_{\mathrm{j}} / \rho_{\mathrm{a}}$, and a single predominantly atomic jet simulation (with a fully molecular ambient medium). In Table 1, we list the values used for these simulations. For each of the light, equal, and dense molecular jet simulations, we employ a grid of $1000 \times 100 \times 100$ uniform zones, and for the atomic jet simulation the grid contained $900 \times 150 \times 150$ uniform zones. In all cases, each zone spanned $2 \times 10^{14} \mathrm{~cm}$ in each direction. The initial jet radius, $R_{\mathrm{j}}$, is $1.7 \times 10^{15} \mathrm{~cm}$ and is therefore resolved by 8.5 zones. With the exception of the zones on the inner $x$-boundary that allow the jet inflow, all of the other boundary zones are set to outflow boundary conditions. In order to make a useful comparison between the dense molecular and the atomic jet simulations, we equate the jet velocity $\left(v_{\mathrm{j}}=100 \mathrm{~km} \mathrm{~s}^{-1}\right)$, rather than the Mach number (reduced from 140 in the molecular jets to 105 in the atomic jet). The Mach number is smaller in the atomic jet owing to the smaller mean molecular weight (more particles).

Detailed tests for shock resolution and numerical convergence were performed by Suttner et al. (1997a). We have improved the resolution from $2.5 \times 10^{14}$ to $2 \times 10^{14} \mathrm{~cm}$, making the present resolution the highest yet achieved for simulations of fully three dimensional stellar jets. A higher resolution simulation, run for a shorter dynamical time, has been executed and demonstrates the same global structures as presented here with some additional fine-scale structure (see also Suttner et al. 1997b).

We also increase the total number of zones by a factor of 3.125. Nevertheless, the grid length of $2 \times 10^{17} \mathrm{~cm}$ is still almost an order of magnitude shorter than the average detected (projected) size of 0.5 pc (Stanke 2000; Eislöffel 2000). A limited size is necessary, however, in order for the physics of individual shocks to be represented. In dissociative shocks, the break up of hydrogen molecules provides the main source of cooling until all the molecules are dissociated. Hence, even with quite large grid sizes, the critical speed at which dissociation occurs can still be approximated. Reformation of molecules is significant in the cool compressed layers behind these hydrodynamic shocks of high Mach number. That is, on the timescale to flow around the bow of $30 \mathrm{yr}$, ten per cent of the molecules can be reformed at the peak density of $10^{7} \mathrm{~cm}^{-3}$ (given the reformation rate of $\sim n 10^{-17} \mathrm{~s}^{-1}$ ). As in other stellar outflow simulations, however, atomic cooling is not resolved in fully-dissociative or atomic shocks. Instead, the time step is limited by restricting the decrease in internal energy in any zone (e.g. Stone \& Norman 1993a). 
The derived emission maps for shock-excited gas should be considered demonstrative and treated with caution. We have sacrificed shock resolution in order to execute simulations in three dimensions. We believe this to be essential since 2D and 3D simulations run under quite similar conditions generate very different global structures (Suttner et al. 1997a). To resolve the atomic cooling layers of shock fronts to sufficient accuracy requires 1000 grid points (Sutherland et al. 2003) spread over a cooling length of order $10^{16} \mathrm{~cm} / n$. We can at least hope to resolve the molecular section of the shock, typically of width $10^{19} \mathrm{~cm} / n$. It is clear that these are only partly resolved here but better resolved in the more dissipative low density medium where the shocks are generally faster. To assume a lower density, however, could resolve the problem provided the cooling length remains much shorter than the bow scale. As shown by Blondin et al. (1990), the flow pattern is only scalable provided this condition is satisfied. However, in molecular flows the cooling length is orders of magnitude longer than the atomic cooling length and this condition becomes difficult to satisfy. The result for a low density molecular jet is that $\mathrm{H}_{2}$ and low-J CO emission is suppressed until the gas has reached the wake of the flow.

The 2D simulations of molecular flows presented by Downes \& Cabrit (2003) study a different regime to that modelled here. They employ a grid size of $1 \times 10^{14} \mathrm{~cm}$ and a jet radius $5 \times 10^{15} \mathrm{~cm}$. Their density of $100 \mathrm{~cm}^{-3}$ implies a molecular cooling length in the $8000 \mathrm{~K}$ to $1000 \mathrm{~K}$ range of $10^{17} \mathrm{~cm}$, far exceeding the bow size. Their results are therefore not scalable to the strongly-cooling molecular flows from Class 0 protostars but would apply to weaker outflows later in the evolution of young stars, provided interstellar UV radiation is weak enough to allow molecule formation.

Since an actual atomic jet would be likely to entrain some molecules as it emerges from the dusty disk and the molecular cloud within which it is embedded, we have set the molecular fraction in the zones partially covered by the inlet to have an averaged (i.e., non-zero) value. This yields an average mean molecular fraction of $0.063(12.6 \%)$ for the inflowing atomic jet, a value that is consistent with 8.5 zones per $R_{\mathrm{j}}$.

We set the unmodified jet velocity, $v_{\mathrm{j}}$, to $100 \mathrm{~km} \mathrm{~s}^{-1}$. All simulated jets are pulsed $\left( \pm 30 \% v_{\mathrm{j}}\right.$, with a $60 \mathrm{yr}$ period), precessed (with a half-opening angle of 1 degree and a $50 \mathrm{yr}$ period), and have a radial shear that reduces the jet velocity at the jet edge to $0.8 v_{\mathrm{j}}$. We show the mass flux, thrust, and mechanical luminosity (power) of the jets in Table 1. We conclude that the dense and atomic jets modelled here would not be able to drive powerful outflows such as Cepheus E but can supply the requirements of intermediate strength outflows such as HH 211 or HH 212.

\section{Internal structure}

These simulations offer an opportunity to compare structures of the underlying physical flow with those that may be observed, for example, by molecular emission lines. The structures of the flow are first displayed here as cross sections of mass density that include views of the midplane (Fig. 1) and normal to the jet axis (Fig. 2).
The midplane density cross sections contain a few notable characteristics:

1. Each of the molecular jets (in the top three panels) possesses a narrow, leading well-focused region beyond some rather squat "shoulders" that span the envelope of the light, warm cocoon.

2. In the atomic jet, the leading region is broader, primarily caused by the less efficient atomic cooling. The relatively thick wedge-like shape of this region typifies atomic jet simulations (e.g., Blondin et al. 1990).

3. The fraction of the length along the jet axis covered by the well-focused region is smaller for simulations with larger $\eta$ (i.e., the jet-to-ambient density ratio is larger).

The midplane cross sections also show the series of high density shocks created by the pulsating jets. The distance between the shocks yields a pattern speed of $\sim 100 \mathrm{~km} \mathrm{~s}^{-1}$, close to the average flow speed. The jet shear leads to a focussing of the flow, with the oblique reverse shocks deflecting the jet towards the axis, while gas overtaken by the forward jet shocks is deflected away from the axis. The result is a jet spine consisting of knots of high momentum. Additionally, we note that a pulsed jet without shear generates lateral knots which move with the average jet speed (run 3D-2 of Suttner et al. 1997a).

Many of these structures are seen in the axial cross sections shown in Fig. 2, such as the extent of the narrow region in both transverse directions. The cross sections that show a corrugated or rough boundary between the bow shock and the ambient medium are located near the leading edge of the shoulder region. The frequency of these rough boundaries is higher in the light jet and atomic jet simulations, indicating that these types of simulations are more susceptible to some instability at this boundary. As with the cross sections in the midplane, the axial cross sections show the internal shocks associated with the pulsing of the jets.

The dense molecular jet crosses the grid in $700 \mathrm{yr}$, the equal dense jet in $850 \mathrm{yr}$, and the light jet in $1400 \mathrm{yr}$. Thus, the ratios of average speeds are $2.0: 1.6: 1.0$ in the dense: equal: light jet simulations. This is a smaller range than the $3.2: 2.1: 1.0$ ratio expected from standard ram pressure arguments (with an assumption that the areas on both sides of the terminal shock are identical). Also, the advance speed of the leading edge of each jet is faster than the expected value by a different amount: the dense jet is only $20 \%$ faster, the equal density jet is $50 \%$, and the light jet is $90 \%$ faster. These different accelerations cause the ratios of the advance speeds to narrow from those expected.

In contrast, the advance speeds of the shoulder regions do obey the ram pressure derived ratios. For each of the molecular jets, the average speed of the shoulder advance is about three quarters of the expected ram pressure advance speed assuming a jet speed of $80 \mathrm{~km} \mathrm{~s}^{-1}$, which corresponds to the inflow speed at the edge $\left(R=R_{\mathrm{j}}\right)$ of the sheared jet. We thus uncover a core/sheath morphology, accentuated by the strong molecular cooling: with the refocused accelerating inner core of the jet surrounded by an abandoned slower moving outer sheath that is advancing at roughly the speed expected from ram pressure arguments. 


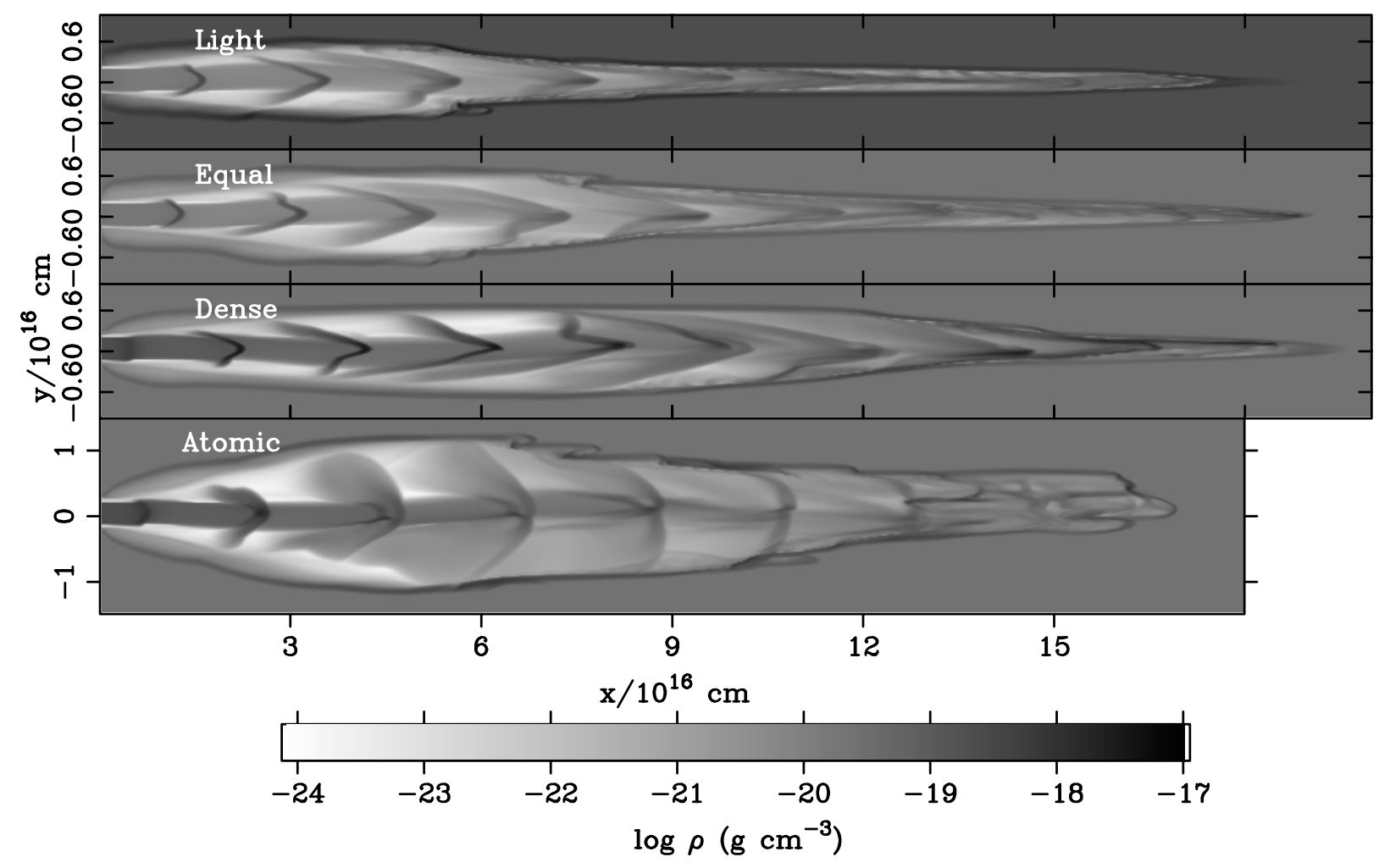

Fig. 1. Midplane cross section of density. The density (in the chosen plane $z / R_{\mathrm{j}}=0.0$ ) for the light jet (top), the equal density jet (second panel), the dense jet (third panel) and the atomic jet (lower panel) at $t=1400,850,700$, and $650 \mathrm{yr}$, respectively, is displayed. Each cross section is scaled to the same maximum and minimum with darker shading indicating denser regions, as quantified below the cross sections. The complete computational domain is represented. The vertical axis is the $y$-axis in all panels.

Alternatively, a mean velocity can be derived from the advance of the bow shock in the simulated emission maps, which we have at a high temporal resolution (dumps every $2 \mathrm{yr}$ ). The results are that the dense molecular jet maintains a mean velocity of about $90-95 \mathrm{~km} \mathrm{~s}^{-1}$ throughout the simulation, the equal density jet has a mean velocity of $80 \mathrm{~km} \mathrm{~s}^{-1}$, and the light jet advances with a typical speed of $50 \mathrm{~km} \mathrm{~s}^{-1}$. The ratio of these speeds is roughly the same as the ratio of speeds derived from grid crossing times. We note that only the atomic jet has a non-steady average speed (based on the advance of the $\mathrm{H}_{2}$ bow shock). In this case, starting at about $t=300 \mathrm{yr}$, the advance speed fell from that of the dense molecular jet, $\sim 90 \mathrm{~km} \mathrm{~s}^{-1}$, to about $70 \mathrm{~km} \mathrm{~s}^{-1}$ by the end of the simulation $(t=650 \mathrm{yr})$. Without the refocusing from the additional molecular cooling, the atomic jet is wider than the molecular one, and can entrain mass and be decelerated more readily.

\section{Simulated molecular line emission}

\subsection{The spatial distributions}

In order to compare the simulations with observations, we have simulated images of the $\mathrm{H}_{2} 1-0 \mathrm{~S}(1), \mathrm{H}_{2} 2-1 \mathrm{~S}(1)$ and CO $0-0 \mathrm{R}(1)$ (i.e., $J=2-1$ ) lines for the light jet (Fig. 3 ), the equal density jet (Fig. 4), the dense jet (Fig. 5), and the atomic jet (Fig. 6). The times are as listed in Fig. 1.

These maps are integrations of line emission over the $z$-axis in the jet, where the line emission is computed from the local characteristics (density, temperature, and molecular fraction) within each zone. The $\mathrm{H}_{2}$ line emission is based on a nonLTE approximation to the vibrational populations (Draine et al. 1983), and the CO emission is from McKee et al. (1982). In addition, since we are only interested in the $\mathrm{CO}$ emission from the jet or entrained material, we integrate the $\mathrm{CO}$ emission only from moving $\left(v>1 \mathrm{~km} \mathrm{~s}^{-1}\right)$ material. Since the appearance of the jets in $\mathrm{H}_{2}$ emission is very similar for both lines that we calculated, and the ratio of the $\mathrm{H}_{2} 2-1$ luminosity to the 1-0 luminosity is related to the local temperature, we show this ratio in the middle panel of Figs. 3-6.

The lower energy rotational $\mathrm{CO}$ emission shows the extent of the accumulated cold mass. The outline of the bow shock is easier to see in the CO line than in either of the higher energy vibrational $\mathrm{H}_{2}$ lines. The latter highlight regions that have been recently strongly shocked. The outline of the bow shock in the individual runs closely reflects the different shapes of the density cross sections in Fig. 1.

In the $\mathrm{H}_{2} 1-0$ line, only the initial internal shock associated with the pulsed jet is apparent for each of the molecular jet simulations. These shocks are more prominent in the atomic jet, where they possess a unique appearance. At least the initial pulse, for some length and time, appears as two pairs of short linear features parallel to, but offset transversely from, the jet axis. These linear features are from integration across the rearward part of the bows.

In the dense jet simulation, the ratio of the $\mathrm{H}_{2} 2-1$ to 1-0 luminosities shows each of the four pulse shocks before 

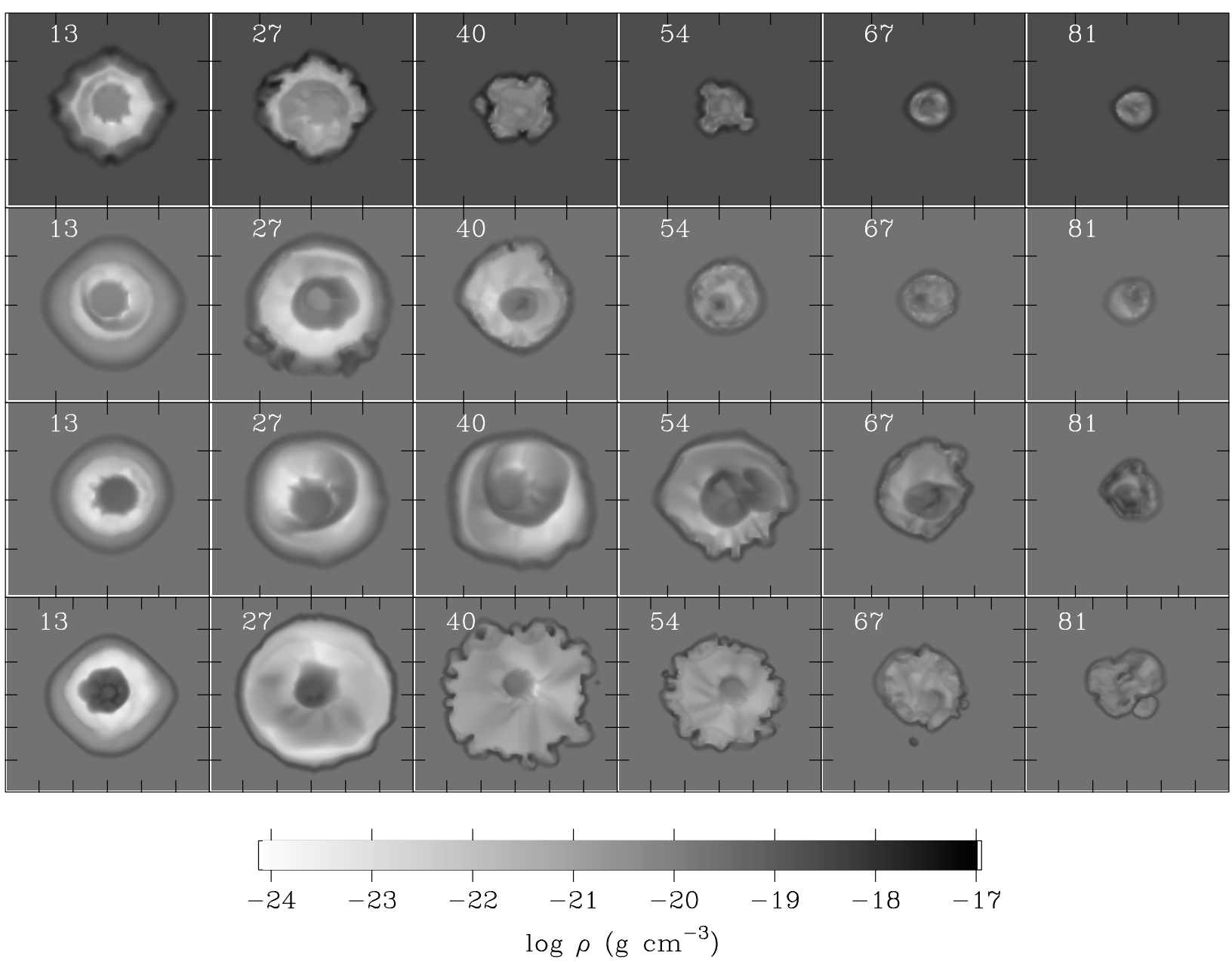

Fig. 2. Axial cross sections of density for the light jet (top row), the equal density jet (second row), the dense jet (third row) and the atomic jet (lower row). The time displayed corresponds to that of the panel in Fig. 1. Each cross section is scaled to the same maximum and minimum and the scale, which has darker shading to indicate denser regions, is displayed underneath. The complete computational domain is shown. The distance between adjacent tick marks is $5 \times 10^{15} \mathrm{~cm}$. Note that the atomic jet simulation spans a larger grid. The axial position in $R_{\mathrm{j}}$ is given in the upper left of each panel. The vertical axis is the $y$-axis, and the horizontal axis is the $z$-axis. So the view is from the jet inlet boundary, looking down the jet axis (i.e., toward the $+x$-direction).

the shoulder region (naturally, plotting this with a dynamic range of 6 orders of magnitude emphasizes these structures). Since the ratio of these line emissions from the light and equal density jet is more uniformly distributed, the internal shocks are less easily seen. This ratioed plot is even more uniform in the atomic jet simulation. In all four simulations, the plots of the ratioed line emissions show that a maximum occurs in the shoulder region, where the jet is at its widest, suggesting that this region has been shock-heated.

The maximum luminosity for the 1-0 line is greatest for the dense molecular jet and reduced by about $0.2-0.4$ dex for the light and atomic jets, with the equal density jet having the lowest maximum luminosity. The lowest maximum in the equal density case, which also occurs in the $\mathrm{CO}$ line, is caused by the low densities $(\log n=4)$ used for both the jet and the ambient medium. The relatively brighter map in the light jet case occurs even with a relatively light jet $\left(\log n_{\mathrm{j}}=4\right)$, since the ambient medium is dense $\left(\log n_{\mathrm{a}}=5\right)$.

The surface of the bow shock of the atomic jet appears very filamentary and has been subject to some instability. This is most apparent in the $\mathrm{H}_{2} 1-0$ line. While this instability appears less well developed in the molecular jets, the very narrow leading refocused region of these jets is not very well resolved. In an unpublished higher resolution simulation (with an earlier version of the molecular cooling function than used in the simulations presented here), we do see evidence for the many "minibows" in the leading part of the dense jet simulation.

\subsection{Totals}

The evolution of the integrated emission for the different emission lines are of great interest to global modeling of star formation (e.g., the Unification Scheme, Smith 2000; Stanke 2000; Yu et al. 2000). These are presented for the four simulations in Fig. 7.

The existence of a factor to convert $\mathrm{H}_{2}$ luminosity into that for the driving jet power would be extremely useful. The ratio of average jet power to the $\mathrm{H}_{2}$ 1-0 emission line at the final recorded times of the simulations are 80 (light jet), 250 (equal density), 400 (dense) and 60 (atomic jet). Thus the dense 


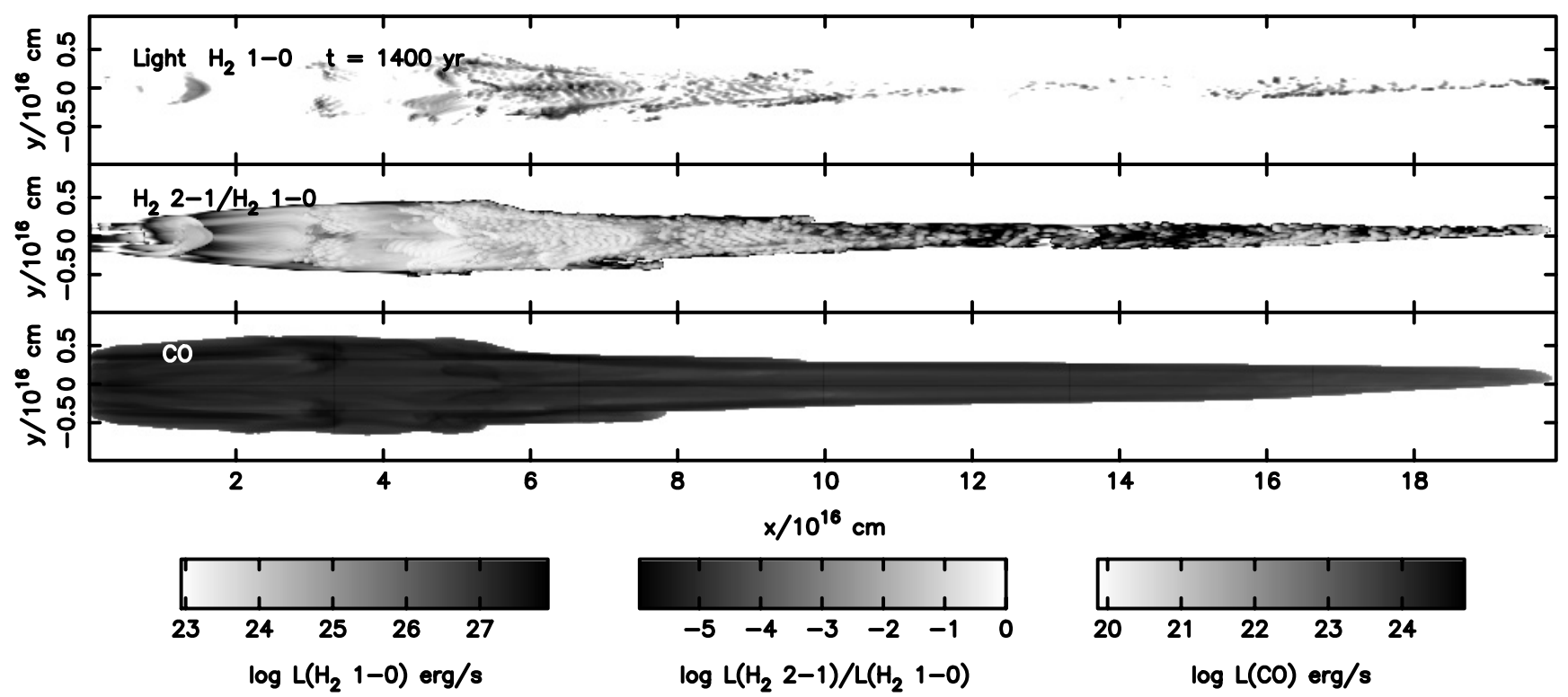

Fig. 3. Images of the light jet simulation in three molecular emission lines. The luminosities from each zone are placed in bins in the viewing window that are roughly the same size as the 3D zones used in the simulations (i.e., $2 \times 10^{14} \mathrm{~cm}$ ). The axis being integrated is the $z$-axis, so the vertical axis in all the panels is the $y$-axis.

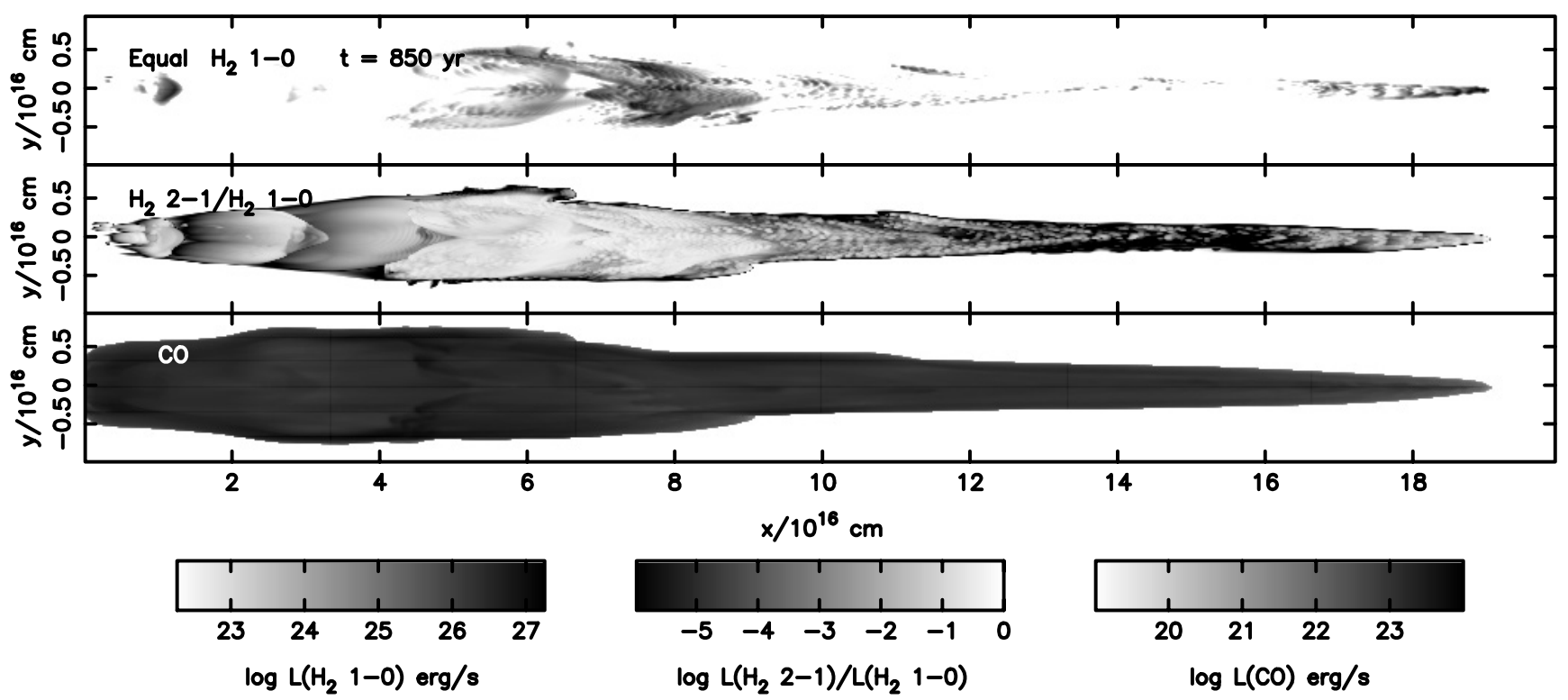

Fig. 4. Images of the equal density jet simulation in three molecular emission lines. See caption to Fig. 3.

molecular jet dissipates energy less efficiently. This is as expected since the leading edge is advancing at high speed. In the classical steady momentum-balance scenario for the impact region, the total energy dissipation rate is

$P=\frac{1}{2} \rho_{\mathrm{j}}\left(U-v_{\mathrm{j}}\right)^{3}+\frac{1}{2} \rho_{\mathrm{a}} U^{3}$

where $U$ is the speed of advance of the leading bow into the ambient medium of density $\rho_{\mathrm{a}}$. Equating thrusts,

$\rho_{\mathrm{j}}\left(U-v_{\mathrm{j}}\right)^{2}=\rho_{\mathrm{a}} U^{2}$,

then yields

$P=\frac{1}{\left(1+\left(\rho_{\mathrm{j}} / \rho_{\mathrm{a}}\right)^{1 / 2}\right)^{2}} \times \frac{1}{2} \rho_{\mathrm{j}} v_{\mathrm{j}}^{3}$.
According to this theory, ballistic jets are less dissipative than light jets, by exactly 10 for the dense and light jet densities considered here. This is roughly consistent with the relative values measured in the molecular jet simulations. The efficiency of the atomic jet rivals that of the light molecular jet. The wider bow shock of the atomic jet causes the greater radiative efficiency. With the wider bow shock, the atomic jet attempts to plough through the ambient medium rather than slip past it, as the narrow molecular jets seem to prefer.

At late times in the simulation, the dense jet has a typical integrated luminosity roughly half an order of magnitude greater than the other molecular jet simulations (in which the initial jet density was one order of magnitude lower, see Table 1). In general, the $\mathrm{H}_{2}$ emission lines appear more constant than 


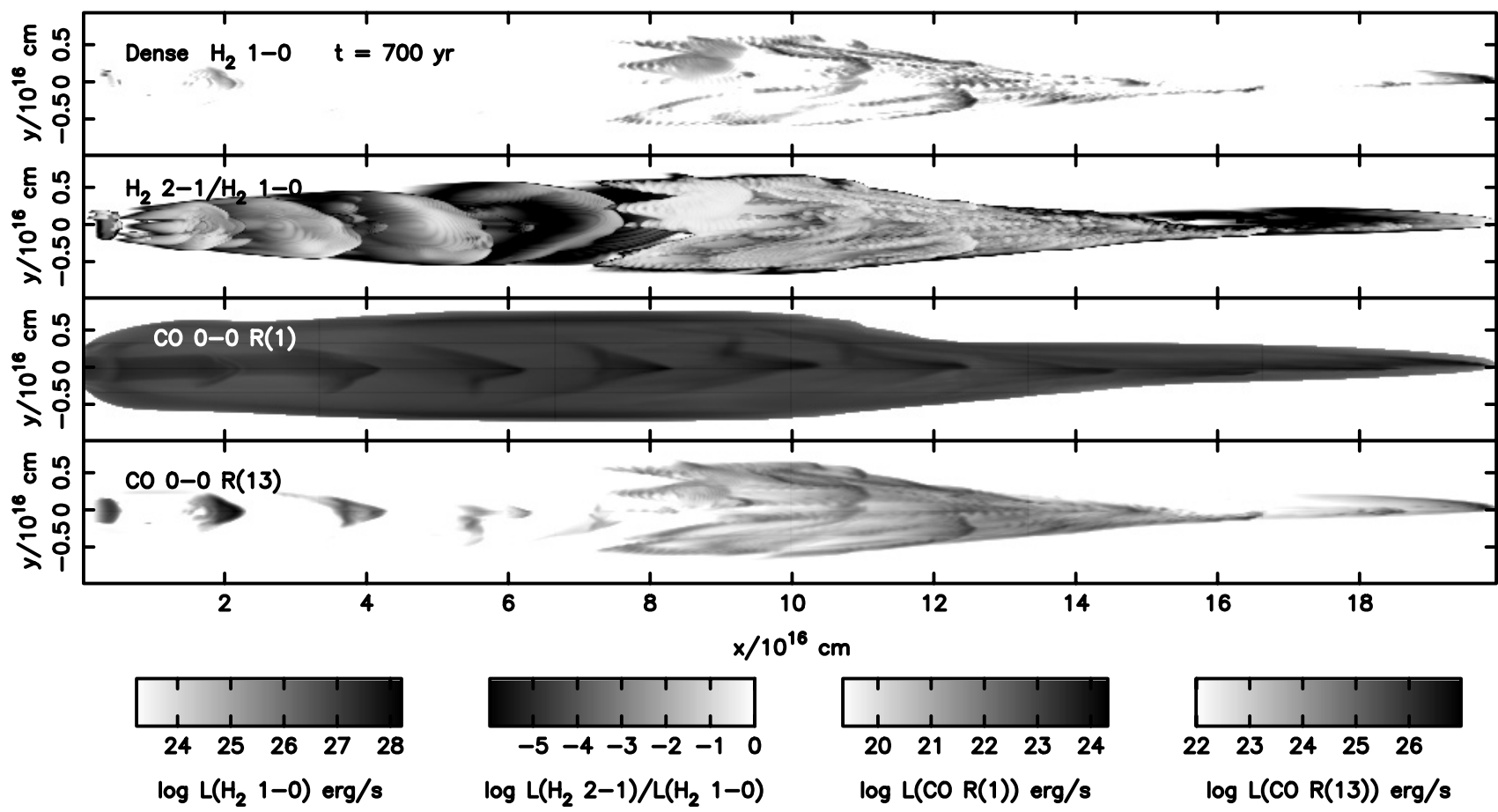

Fig. 5. Simulated images of the dense jet run in three molecular emission lines. See caption to Fig. 3. The lower panel shows the estimated emission for CO $J=14-13$ emission, predicting the appearance at $186 \mu \mathrm{m}$ that could be observed by the Herschel/FIRST mission (discussed in Sect. 6).

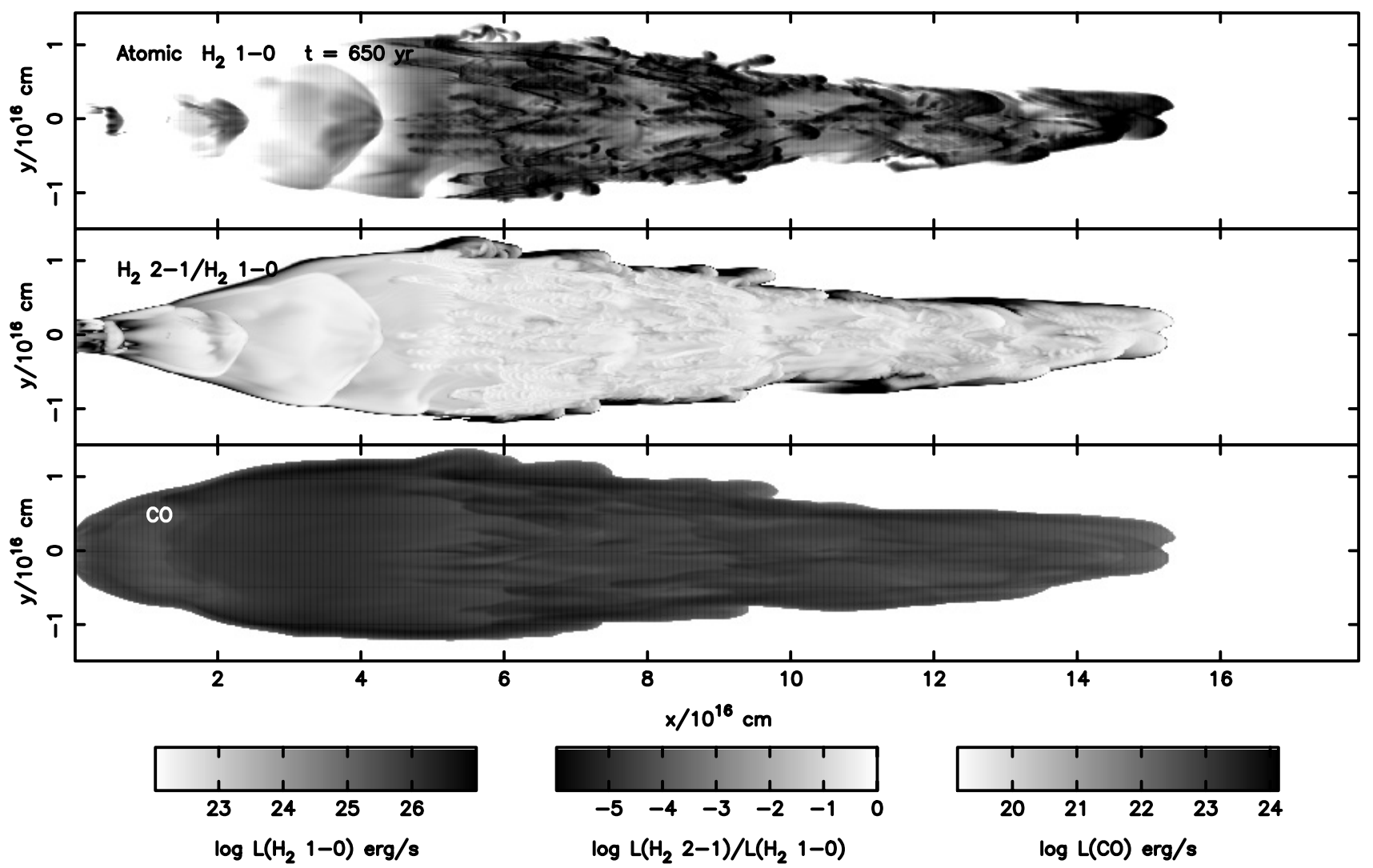

Fig. 6. Images of the atomic jet simulation in three molecular emission lines. See caption in Fig. 3.

the CO line emission, which increases over time and reflects the accumulation of colder mass in a region consumed by an expanding bow shock. However, there are two exceptions of note. First, the integrated emission in $\mathrm{H}_{2}$ undergoes the greatest change in the atomic jet simulation, where the $\mathrm{H}_{2}$ also increases with time as the $\mathrm{CO}$ does (but to a lesser extent). Second, the 


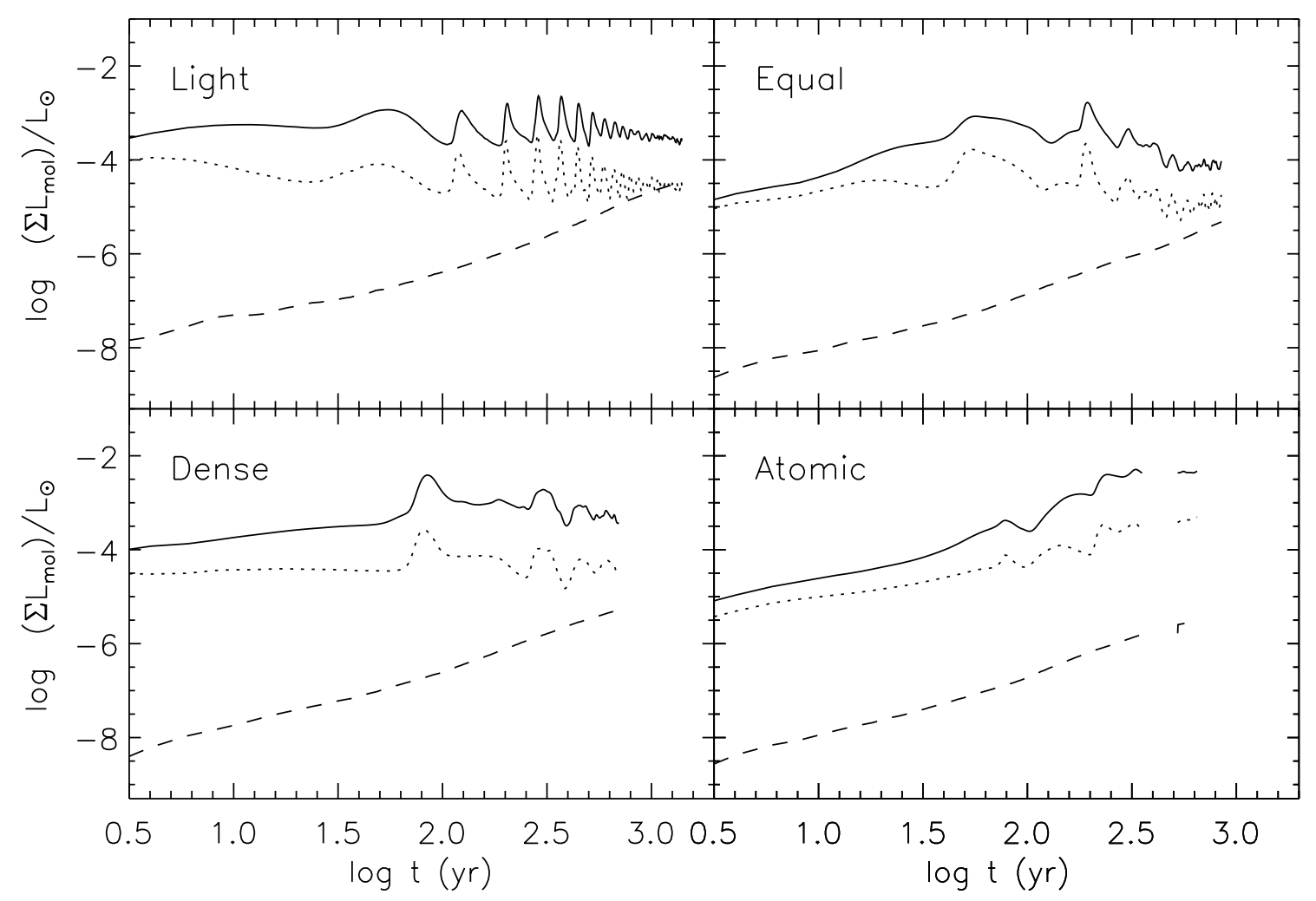

Fig. 7. Integrated molecular emission in three lines for each simulation. The $\mathrm{H}_{2}$ 1-0 emission (solid line), the $\mathrm{H}_{2} 2-1$ (dotted line) and the $\mathrm{CO}$ emission (dashed line) are displayed.

molecular jet simulations, but particularly the light jet simulation, exhibit short term brightenings in total $\mathrm{H}_{2}$ emission (either line measured here). We show in a time sequence of the 1-0 emission (Fig. 8) that these quick brightenings are the result of a pulse passing the main "shoulder" of material just before the refocused region. These pulses periodically light up the shoulder region.

\section{Velocity distribution of mass and molecular line emission}

\subsection{Mass- and line emission luminosity-velocity relation}

One currently common way to present $\mathrm{CO}$ velocity data is to plot the differential mass in velocity bins, and then to see how this mass decreases with velocity, i.e. $\mathrm{d} m / \mathrm{d} v \propto v^{-\gamma}$. The mass is derived from the $\mathrm{CO}$ intensity by assuming optically thin emission, a fixed temperature and a fixed abundance. In these simulations, we only make the first assumption.

In at least two surveys of many sources (Yu et al. 2000; Ridge \& Moore 2001), the analysis leads to a broken power law, i.e. a shallow slope of 1.0-2.0 at low velocities and a steeper slope, in some cases $\gamma$ is close to 10 , at larger velocities. The velocity where the break occurs is usually $\sim 10 \mathrm{~km} \mathrm{~s}^{-1}$. One interpretation of this break speed is that it is the projected component of the critical shock speed, $v_{\mathrm{d}} \cos \theta$ (Downes \& Cabrit 2003) with $v_{\mathrm{d}}=23 \mathrm{~km} \mathrm{~s}^{-2}$ (e.g., Smith 1994), above which all molecules are assumed to dissociate within a shock.
Downes \& Cabrit (2003) make a laudable attempt to analyze a few simulations for mass-velocity and molecular line intensity-velocity relationships. However, the $\mathrm{CO}$ line emission approximations from McKee et al. (1982) that they employ are only valid for number densities above $\gtrsim 10^{4}$, larger than exists in many (but not all) zones in their simulations.

We have performed a similar analysis, also using the approximations of McKee et al. (1982), on each of the four jet simulations for different viewing angles. We define the viewing angle as the angle of the jet axis relative to the plane of sky and toward the observer (so most velocities are negative for non-negative viewing angles). We estimated $\gamma$ for the mass as well as emission from the $\mathrm{CO}$ line and $\mathrm{H}_{2}$ 1-0 line shown previously (using the same emission line prescriptions as employed for Figs. 3-6). We have used a linear fitting routine over a subset of the velocities to determine the values for $\gamma$. The estimates for the fall-off from the mass and $\mathrm{CO}$ emission are listed in Table 2 for many viewing angles and shown for one $\left(15^{\circ}\right)$ in Fig. 9.

Perhaps the most important result here is that $\gamma_{\text {mass }}$ is consistently lower than $\gamma_{\mathrm{CO}}$ in all simulations. For the dense and atomic jet simulations the difference was $\sim 0.3$, but this was reduced for the light and equal density simulations. Also, this difference is largest for the smallest of the viewing angles that we investigated here, and the difference decreases with viewing angle. Downes \& Cabrit (2003) find a similar difference at high velocities, with swept up mass (they include a tracer that follows the jet material and have excluded it) having a shallower fall off than that in $\mathrm{CO}$ intensity. 


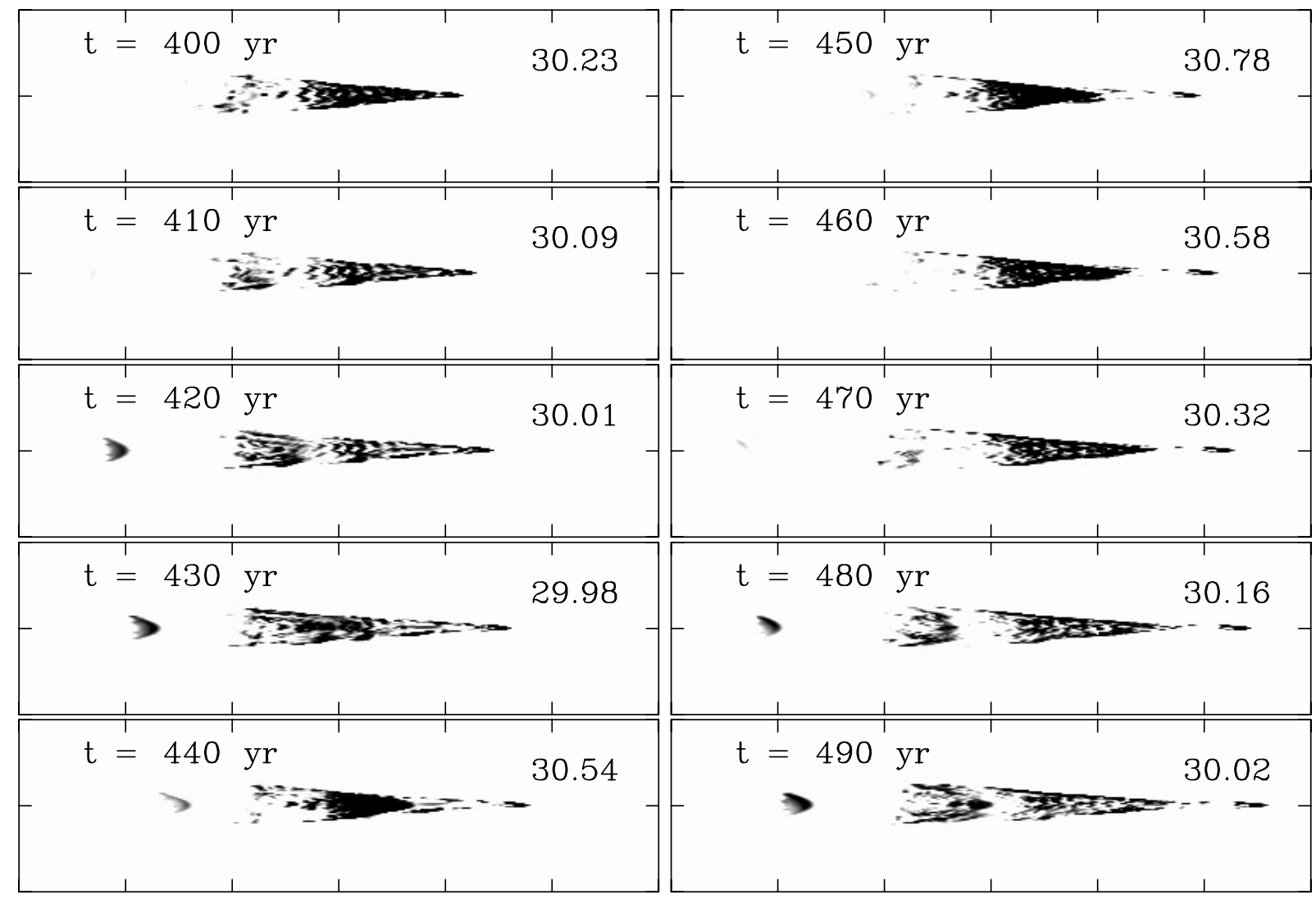

Fig. 8. A pulse traversing and brightening the shoulder region in the light jet. The progression of a pulse-generated shock as it approaches and passes through the leading bow shock is easily traced in the $\mathrm{H}_{2} 1-0$ line. As it propagates through the already emitting bow shock region, the pulse adds its energy to the shocked gas and illuminates the nearby regions. This sequence corresponds to one of the brightening episodes in Fig. 7. The view of each image spans $2 \times 10^{16} \mathrm{~cm}$ by $6 \times 10^{16} \mathrm{~cm}$, and includes the jet inlet at the left side.

Table 2. Mass spectra power-law dependences.

\begin{tabular}{|c|c|c|c|c|c|c|c|c|c|c|c|}
\hline Run & $t(\mathrm{yr})$ & type & $\theta$ & range of $\log v$ & $\gamma$ & $N^{a}$ & type & $\theta$ & range of $\log v$ & $\gamma$ & $N^{a}$ \\
\hline \multirow[t]{5}{*}{ Light } & \multirow[t]{5}{*}{1400} & \multirow[t]{5}{*}{ mass } & 15 & $0.5-1.0$ & 1.98 & 7 & \multirow[t]{5}{*}{$\mathrm{CO}$} & 15 & $0.5-1.0$ & 2.16 & 7 \\
\hline & & & 30 & $0.5-1.0$ & 1.78 & 7 & & 30 & $0.5-1.0 / 1.0-1.2$ & $1.86 / 2.93$ & $7 / 6$ \\
\hline & & & 45 & $0.5-1.0$ & 1.80 & 7 & & 45 & $0.5-1.0$ & 1.90 & 7 \\
\hline & & & 60 & $0.5-1.0$ & 1.83 & 7 & & 60 & $0.5-1.0 / 1.2-1.4$ & $1.95 / 2.80$ & $7 / 9$ \\
\hline & & & 90 & $0.5-1.0$ & 1.79 & 7 & & 90 & $0.5-1.0 / 1.3-1.5$ & $1.96 / 2.93$ & $7 / 12$ \\
\hline \multirow[t]{5}{*}{ Equal } & \multirow[t]{5}{*}{850} & \multirow[t]{5}{*}{ mass } & 15 & $0.3-0.7 / 0.5-1.0$ & $2.20 / 1.60$ & $3 / 7$ & \multirow[t]{5}{*}{$\mathrm{CO}$} & 15 & $0.3-0.7 / 0.5-1.0$ & $2.35 / 1.73$ & $3 / 7$ \\
\hline & & & 30 & $0.5-1.0$ & 1.73 & 7 & & 30 & $0.5-1.0$ & 1.77 & 7 \\
\hline & & & 45 & $0.5-1.0$ & 1.63 & 7 & & 45 & $0.5-1.0$ & 1.73 & 7 \\
\hline & & & 60 & $0.5-1.0$ & 1.53 & 7 & & 60 & $0.5-1.0$ & 1.68 & 7 \\
\hline & & & 90 & $0.5-1.0$ & 1.46 & 7 & & 60 & $0.5-1.0$ & 1.63 & 7 \\
\hline \multirow[t]{5}{*}{ Dense } & \multirow[t]{5}{*}{700} & \multirow[t]{5}{*}{ mass } & 15 & $0.3-0.7 / 0.9-1.0$ & $0.78 / 1.53$ & $7 / 2$ & \multirow[t]{5}{*}{$\mathrm{CO}$} & 15 & $0.5-1.0$ & 1.29 & 7 \\
\hline & & & 30 & $0.5-1.0 / 1.2-1.4$ & $0.79 / 1.40$ & $7 / 9$ & & 30 & $0.5-1.0 / 1.1-1.4$ & $1.09 / 1.28$ & $7 / 12$ \\
\hline & & & 45 & $0.5-1.0 / 1.3-1.5$ & $0.70 / 1.42$ & $7 / 12$ & & 45 & $0.5-1.0 / 1.1-1.5$ & $0.95 / 1.28$ & $7 / 19$ \\
\hline & & & 60 & $0.5-1.0 / 1.3-1.5$ & $0.68 / 1.25$ & $7 / 12$ & & 60 & $0.5-1.0$ & 0.93 & 7 \\
\hline & & & 90 & $0.5-1.0 / 1.5-1.7$ & $0.67 / 1.26$ & $7 / 18$ & & 90 & $0.5-1.0$ & 0.93 & 7 \\
\hline \multirow[t]{5}{*}{ Atomic } & \multirow[t]{5}{*}{650} & \multirow[t]{5}{*}{ mass } & 15 & $0.2-0.7 / 0.7-1.1$ & $0.69 / 1.51$ & $3 / 8$ & \multirow[t]{5}{*}{$\mathrm{CO}$} & 15 & $0.2-0.7 / 0.7-1.1$ & $1.18 / 2.31$ & $3 / 8$ \\
\hline & & & 30 & $0.4-0.9 / 1.1-1.4$ & $0.78 / 1.70$ & $5 / 12$ & & 30 & $0.4-0.9 / 1.1-1.4$ & $1.06 / 2.57$ & $5 / 12$ \\
\hline & & & 45 & $0.5-1.0 / 1.4-1.6$ & $0.65 / 1.95$ & $7 / 15$ & & 45 & $0.5-1.0 / 1.4-1.6$ & $0.90 / 3.24$ & $7 / 15$ \\
\hline & & & 60 & $0.5-1.0 / 1.5-1.7$ & $0.55 / 2.09$ & $7 / 18$ & & 60 & $0.5-1.0 / 1.5-1.7$ & $0.76 / 3.50$ & $7 / 18$ \\
\hline & & & 90 & $0.5-1.0 / 1.5-1.7$ & $0.53 / 1.95$ & $7 / 18$ & & 90 & $0.5-1.0 / 1.5-1.7$ & $0.76 / 2.78$ & $7 / 18$ \\
\hline
\end{tabular}

${ }^{a} N$ is the number of points in the velocity distribution, which was computed in $1 \mathrm{~km} \mathrm{~s}^{-1}$ bins, used to estimate the slope. 


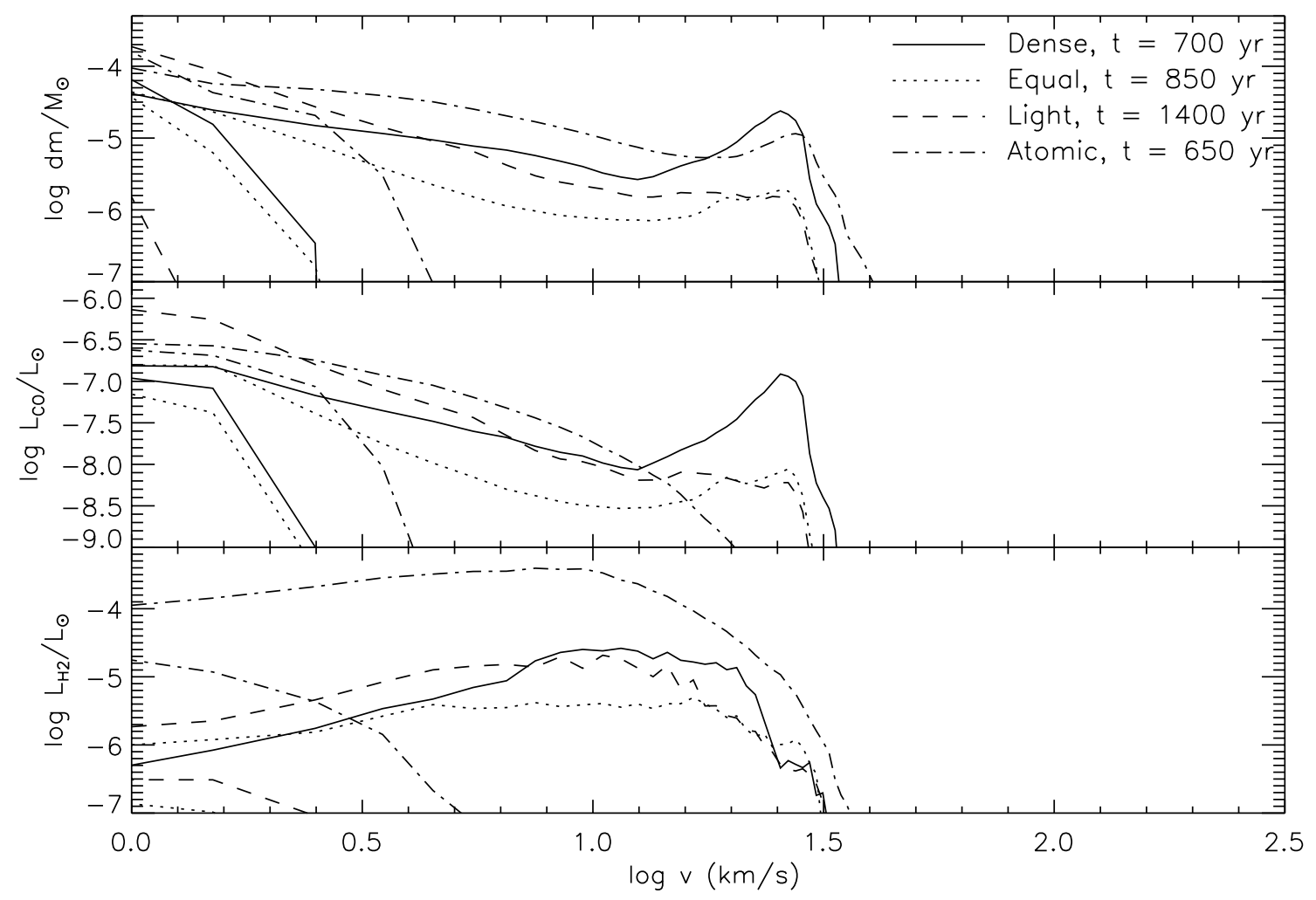

Fig. 9. Distribution of velocities into bins of mass and two molecular line luminosities for a viewing angle of $15^{\circ}$. The distribution of mass (top), $\mathrm{CO}$ luminosity (middle) and $\mathrm{H}_{2} 1-0$ emission (lower panel) are displayed. Each velocity bin is $1 \mathrm{~km} \mathrm{~s}^{-1}$ wide. The line style for the data presented in each panel is shown in the top panel. Most of the line styles are represented twice within each panel, with data from both positive and negative radial velocities included. Naturally, the smaller ranged data is for the positive radial velocities (which could contribute to a red-shifted lobe, while the other is from a blue-shifted lobe).

In general, the $\gamma$ for mass or $\mathrm{CO}$ decreases with increasing inclination angle, as recognised from previous simulations (Smith et al. 1997; Lee et al. 2001; Downes \& Cabrit 2003). However, there are exceptions to this, with more deviations for the distributions in $\mathrm{CO}$, for lower densities, or for the $\gamma$ derived in the high velocity range.

The fall-off with velocity becomes shallower with jet-toambient density ratio, although the difference between $\gamma_{s}$ in the equal density and light jet cases are smaller than those between the equal density and dense jet cases. Some of this may result from using the same jet density for the equal density and light jet case while the dense jet had a larger jet density than both.

Even though the dense molecular jet simulation and the atomic jet (also with an $\eta=10$ ) have similar small-velocity slopes, there is a caveat for those in the atomic jet. For the atomic jet, some of the mass- and CO intensity-velocity distributions are not well represented by power-laws. In addition, the high velocity bump that is present in mass in the atomic case and in the $\mathrm{CO}$ distribution in all the other cases, is absent in the $\mathrm{CO}$ distribution in the atomic jet.

Some of these characteristics are also seen in the $\mathrm{CO}$ intensity-velocity relation in the pulsed jet simulation of Downes \& Cabrit (2003) (their Fig. 2). As with our atomic jet, the CO intensity-velocity relation shown does not seem well-fit by a power law for large ranges of velocity, but appears closer to an exponential law. Also, at low velocities their intensity converts to a luminosity of $10^{-8} L_{\odot}$. This is a factor of 30 below our low-velocity luminosity for the atomic simulation, possibly consistent with their lower density. We thus conclude that further work is necessary before we can consider that numerical simulations are able to predict more than the basic features.

In comparison with observations, the slopes of our velocity distributions are lower than the typical values, which are around 2. However, we note that Ridge \& Moore (2001) state that the larger values for $\gamma$ are associated with outflows from high-mass star formation. Given the mechanical luminosity of our simulated jets (see Table 1), it is then not surprising that our slopes are small. In fact, the $\gamma$ for the dense molecular jet at large viewing angles $\left(>45^{\circ}\right)$ as determined from the $\mathrm{CO}$ emission is remarkably similar to the results of Ridge \& Moore for both the red and blue lobes of NGC 6334I. In this source, the lobes are overlapping and vibrationally-excited $\mathrm{H}_{2}$ possesses high radial speed (Eislöffel et al. 2000), both suggesting jets orientated well out of the sky plane and, therefore, consistent with our results.

In addition to analysing $\mathrm{CO}$ observational data in this fashion, recent observations of $\mathrm{H}_{2}$ emission from protostellar outflows have been similarly analysed (Salas \& Cruz-González 2002). As with the $\mathrm{CO}$ data, the $\mathrm{H}_{2}$ data show a break in behaviour between a flat or slightly rising flux at low velocities and a fall-off in flux with $\gamma$ in the range between 1.8 and 2.6. The break velocity is between $2-17 \mathrm{~km} \mathrm{~s}^{-1}$ for the selection of sources that they examined. From Fig. 9 here, we note that the atomic jet simulation most closely matches the $\mathrm{H}_{2}$ 


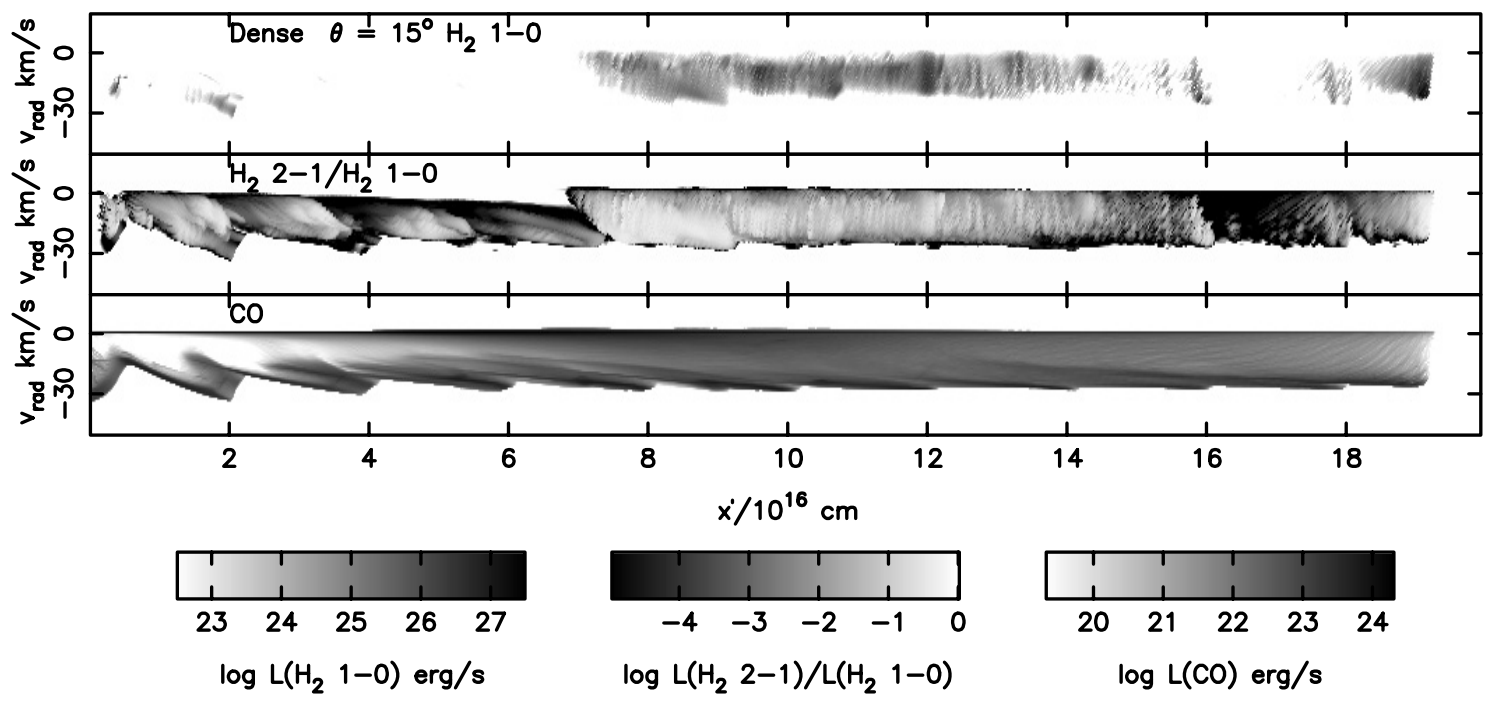

Fig. 10. Sample position velocity map for the dense molecular jet simulation in three molecular emission lines. The jet axis is $15^{\circ}$ out of the plane of the sky toward the observer. Each bin spans $2 \times 10^{14} \mathrm{~cm}$ in $x^{\prime}$, the jet axis projected onto the plane of the sky, and $1 \mathrm{~km} \mathrm{~s}^{-1}$ in radial velocity.

observations. However, as with the $\mathrm{CO}$ velocity distribution for the atomic jet, the fall-off in the $\mathrm{H}_{2}$ velocity distribution differs from a strict power-law. On the other hand, the velocity distribution of the $\mathrm{H}_{2}$ emission in the molecular jet simulations rises fast at small velocities and falls off quickly at larger velocities. Only one example from the Salas \& Cruz-González sample, HH 211, possesses comparable properties.

The shape of line profiles from molecular hydrogen depends on the shock physics since the emission is produced from warm gas. Here, we are limited to simulating J-type hydrodynamic shocks in which the radiation is produced after the shock front compression. Steady-state models of J-type bows then demonstrate, as found here, peaks in intrinsic profile shapes (Smith et al. 2003). C-shock physics applies when the shock viscosity is provided by ambipolar diffusion. This has two potentially off-setting effects: strong emission arises before much shock compression (due to ion-neutral friction) and much higher shock speeds are possible before dissociation of the molecules occurs. Models of steady-state bow shocks demonstrate that the overall result is that $\mathrm{H}_{2}$ emission from C-type bows is predominantly generated from low-speed material, with no peak at intermediate speeds (Smith et al. 2003).

However, Salas \& Cruz-González (2002) point out that the linear fall-off of $\mathrm{H}_{2}$ flux with $\log v$ is only maintained for $v$ less than roughly $40 \mathrm{~km} \mathrm{~s}^{-1}$. Above this, the distribution can become more shallow or steepen. Additionally, within the higher range of $v$, a peak in the distribution is detected from a couple of sources (OMC-1 and HH 211). Not too dissimilar peaks are seen in the $\mathrm{H}_{2}$ distributions in Fig. 9 for all the molecular jet simulations, albeit at very low luminosities.

Downes \& Cabrit (2003) obtain a quite good match for the slope for a $\mathrm{H}_{2}$ intensity-velocity relation. However, even the authors themselves point out that the moderate densities achieved in their simulations do not fully justify the simple assumptions (i.e., LTE) made for computing the line emissivities. Given that the low resolution of our simulations may underestimate the temperatures in many zones and additional physics will be needed to fully model the $\mathrm{H}_{2}$ intensity-velocity relation, we advise caution in the interpretation of either their results or our own for this relation.

\subsection{Position-velocity diagrams: Hubble laws}

We present a position-velocity plot for the dense molecular jet simulation for emission in $\mathrm{H}_{2}$ and $\mathrm{CO}$ (see Fig. 10). The initial pulses are not seen in the $\mathrm{H}_{2}$ maps, which primarily show the leading parts of the bow shock and shoulder region. Note that the narrow shocks generate vertical bars on this diagram. In contrast, the $\mathrm{CO}$ map shows a sequence of Hubble Law-like regions associated with each pulse, most easily seen at small $x$. Of note here is a connecting loop of $\mathrm{CO}$ emission between each of these separate regions, a signature of the precession of the initial jet flow. Moreover, these connecting loops are more noticeable in jets with larger angles of precession (as will be reported in future work by the authors).

Similar position-velocity maps for the other simulations show a few interesting results: 1) since the greatest $\mathrm{H}_{2}$ emission originates from the leading parts of both the highly focused region and the shoulder region, the $\mathrm{H}_{2}$ position-velocity maps for the molecular simulations show that the spacing between these two bright regions increases as $\eta$ decreases (from dense to equal to light). 2) The atomic jet simulation produces a more filled-in $\mathrm{H}_{2}$ position-velocity map, just as it did for the integrated emission maps (compare Figs. 5 and 6). 3) The dense jet simulations contain the brightest bin of $\mathrm{H}_{2}$ emission. 4) In contrast, the light molecular jet simulation has the brightest bin of $\mathrm{CO}$ emission. As with the simulated emission maps of $\mathrm{CO}$ in Figs. 3-6, we impose a minimum velocity cut-off for the position-velocity maps. However, the brightest bin of CO emission is usually within a feature that spans the length of the jet at small velocities. When combined with the channel maps described below, we see that this bright $\mathrm{CO}$ emission is related 


\begin{tabular}{|c|c|}
\hline-35.0 & 21 \\
\hline 33.0 & 22.32 \\
\hline 31.0 & $\cdot \cdot$ \\
\hline 29.0 & $\therefore-\cdots-\cdots-\cdots$ \\
\hline .0 & $\therefore--a-1$ \\
\hline 0 & $\rightarrow \infty-2 \Rightarrow$ \\
\hline & nendes 22 \\
\hline .0 & and 22.57 \\
\hline-19.0 & "nnDD2 22.49 \\
\hline-17.0 & xา 22.51 \\
\hline-15.0 & 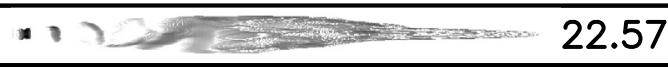 \\
\hline-13.0 & $\cdots 22.32$ \\
\hline-11.0 & 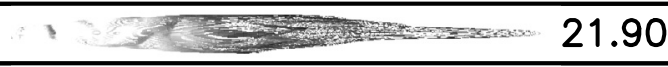 \\
\hline-9.0 & 21.94 \\
\hline-7.0 & 22.11 \\
\hline-5.0 & 22.29 \\
\hline-3.0 & 22.26 \\
\hline-1.0 & 22.59 \\
\hline 1.0 & 22.51 \\
\hline 3.0 & 21.62 \\
\hline
\end{tabular}

Fig. 11. Sample channel map for the dense jet simulation. The jet is viewed $15^{\circ}$ out of the plane of the sky toward the observer. The number on the left of the panel is the central radial velocity, and on the right is the log of the maximum luminosity in ergs $\mathrm{s}^{-1}$. Each bin spans $2 \times 10^{14} \mathrm{~cm}$ in $x^{\prime}$, the jet axis projected on the plane of the sky, and in $y$.

to the sweeping up of the dense ambient medium by the bow shock. 5) In the CO position-velocity maps of the atomic jet simulation, the Hubble-Law regions are less obvious and the regions are not connected (by looping structures or otherwise).

\subsection{Velocity channel map}

We present velocity channel maps in $\mathrm{CO}$ in Fig. 11 for a viewing angle of $15^{\circ}$, again for the dense molecular jet simulation. At high (negative) velocities, much of the internal structure and, specifically, the internal shocks associated with the jet pulses are apparent. At radial velocities near zero, the full extent of the jet plus the surrounding cocoon is identifiable. This morphological difference is similar to that observed in CO emission from protostellar sources (e.g., HH 211, Gueth \& Guilloteau 1999; HH 288, Gueth et al. 2001). Note the length of the gap between the source and the beginning of the $\mathrm{CO}$ outflow. The linear velocity dependence of this gap length is another example of the Hubble-like features in the positionvelocity diagram. More detailed structure is also predicted, and will be described below.

\section{Summary}

We have presented results from a sequence of molecular jet simulations that vary in jet-to-ambient density ratio, as well as an atomic jet simulation with the equivalent hydrogenic nucleon number density and velocity of the dense molecular jet. These are also the first fully 3D simulations of highly collimated jets with velocity shear. The main results are:

1. The molecular cooling and shear combine to help refocus the leading inner part of the jet, so that the front edge of the bow accelerates away from the rest of the flow. The shear that we have included in the jets leads to "shoulders" of material that the accelerating bow leaves behind. Shoulder structures similar to those simulated here have been observed in the W75N outflow (Davis et al. 1998), L1634 (Lee et al. 2000) and HH 195 (Eislöffel 2000).

2. The shoulders move forward at a speed that is proportional to the speed of advance expected from simple ram pressure arguments, so the position of the shoulders relative to the leading edge of the bow is dependent on the jet-to-ambient density ratio.

3. The leading part of the bow in an equivalent atomic jet does not show the amount of refocusing or acceleration that we see in the molecular jets. Additionally, the entire surface of the bow appears dynamically unstable, with many corrugations seen in cross sections taken transverse to the jet axis (Fig. 6). In this respect, the DR 21 outflow appears remarkably similar in $\mathrm{H}_{2}$ to that shown in Fig. 6 (Davis \& Smith 1996). Coincidently, DR 21 is believed to be driven by a jet of atomic H (Russel et al. 1991).

4. The simulated images in $\mathrm{H}_{2}$ lines display some but not all of the bow shock structure, with only small strongly shocked "minibows". Isolated fast-moving bow shocks, capable of propagating large distances, are formed. Comparable examples of extreme collimation are HH111/113/311 (Reipurth et al. 1997), the $\mathrm{H}$ and $\mathrm{N}$ outflows from CB 34 (Khanzadyan et al. 2002), and the parsec-scale outflow from IRAS 05399-0121 (Bally et al. 2002).

5. Internal shocks from the pulsing of the molecular jets are more readily seen in the cross sections of density than in the $\mathrm{H}_{2}$ line emission, where they disappear for certain intervals of time, only to reappear.

6. The CO emission maps from moving material in the simulations shows the overall shape of the jet and shocked ambient gas that is within the bow shock.

7. The total luminosity from the $\mathrm{H}_{2} 1-0$ line confirms that it is equal to roughly $1 \%$ of the input jet mechanical luminosity. We also see that the conversion from jet power to $\mathrm{H}_{2}$ emission is more efficient for the lighter jets, which are associated with slow bow shock advance speeds. Only in the atomic jet did the total $\mathrm{H}_{2}$ line emission increase steadily over time. In all of the simulations, the $\mathrm{CO}$ line emission increased consistently over time.

8. The velocity distribution for mass and the one derived through the estimated $\mathrm{CO}$ emission differ. Similar to a result for the lower jet density simulations of Downes \& Cabrit (2003), we found $\gamma_{\text {mass }}$ to be consistently lower than $\gamma_{\mathrm{CO}}$. For the dense and atomic jet simulations the 
difference was $\sim 0.3$, but this was reduced for the light and equal density simulations.

9. While the values for $\gamma$ from the simulations are typically lower than the most commonly found values from observations of 1.5-2.0, there are some sources that have been fit with $\gamma$ as low as 1.0 (e.g., both the red and blue lobes of NGC 6334I, Ridge \& Moore 2001).

10. We also derive velocity distributions for $\mathrm{H}_{2}$ emission with the aim of comparing our results with those of Salas \& Cruz-González (2002). The comparison is less than compelling with the exception of the HH 211 outflow. We do not find a flat distribution at low velocities and at high velocities the simulated distributions usually decrease nonlinearly with $\log v$. We believe this problem could be solved by considering magnetohydrodynamic processes within the cooling layers and applying ambipolar diffusion to soften the shock acceleration.

11. We also show examples of position-velocity and velocity channel maps in $\mathrm{CO}$ emission. In the former for the molecular jets, we see connected looping structures than connect the Hubble-Law like regions associated with each jet pulse. In the velocity channel maps, we note internal structure at high velocities and the jet and surrounding cocoon at low velocities, not unlike observations.

Jets from the youngest low mass Class 0 protostars drive molecular outflows that are up to an order of magnitude more powerful than their equivalent Class 1 counterparts. It may simply be that Class 0 jets are more powerful. Alternatively, the simulations presented here suggest that, given the same environment, Class 0 jets could be lighter and therefore more efficiently radiative than Class 1 jets rather than more powerful.

ALMA will measure fine-scale spatial variations in $\mathrm{CO}$ emission. The internal structure as well as the distribution of the bullets formed within jets will be of immense interest and enable us to understand the molecular physics of jets. As demonstrated in these high-collimation simulations, a chain of bullets that merge into a continuous stream is predicted at high radial velocity in low rotational CO lines (Fig. 11). A flat-fish spinal structure is predicted at moderate velocities. At low speeds, material may accumulate behind mini-bow shocks along the shell, producing flow-aligned linear filamentary structure (most notable in an outflow driven by an atomic jet). The location of the shoulders and the size of the gap between the source and the outflow at moderate speeds will also severely test the model.

Herschel/FIRST will have the unique capabilities of performing photometry and medium resolution spectroscopy in the 80-600 micron band. This includes many rotational CO lines, including that corresponding to the CO $J=14-13$ transition at $186 \mu \mathrm{m}$, which can be measured with the bolometric array camera PACS on the Herschel satellite. We predict the appearance of bow-shaped bullets and strong emission from the shoulders, generally much sharper structures than in the submillimetre and millimetre CO lines (e.g., the bottom panel in Fig. 5).

Can we relate the properties of a sequence of outflows driven by jets of decreasing density to the evolution of the driving protostar, with dense jets corresponding to Class 0 outflows? These simulations of highly collimated jets suggest not, mainly because refocussing leads to quite similar structure provided the jet is molecular. Moreover, dense jets are the least efficient in dissipating jet energy via the 1-0 S(1) near-infrared line whereas, observationally, this channel appears to be the most efficient (in comparison to the bolometric luminosity of the driving source) during the Class 0 stage (Stanke 2000; Froebrich et al. 2003).

In other work, we have investigated the differences between evolving protostellar jets (increasing vs. decreasing mass flux) (Rosen \& Smith 2003). In the near future, we will examine the influence of precession angle on both the physical and derived quantities presented in this paper. The influence of a magnetic field and a non-uniform ambient cloud can also be elucidated on comparison to the present basic study.

Acknowledgements. The numerical calculations were run on the local SGI Origin 2000 computer (FORGE), acquired through the PPARC JREI initiative with SGI participation. AR is funded by PPARC. The unpublished higher resolution simulation mentioned in Sect. 4 was run on an SGI 3000 at the UK Astrophysical Fluids Facility (UKAFF). We thank the anonymous referee for raising several issues.

\section{References}

Bachiller, R. 1996, ARA\&A, 34, 111

Bally, J., \& Lada, C. J. 1983, ApJ, 265, 824

Bally, J., Reipurth, B., \& Aspin, C. 2002, ApJ, 574, L79

Beuther, H., Schilke, P., Gueth, F., et al. 2002, A\&A, 387, 931

Blondin, J. M., Fryxell, B. A., \& Konigl, A. 1990, ApJ, 360, 370

Bontemps, S., Andre, P., Terebey, S., \& Cabrit, S. 1996, A\&A, 311, 858

Cerqueira, A. H., \& de Gouveia dal Pino, E. M. 1999, ApJ, 510, 828

Cerqueira, A. H., \& de Gouveia Dal Pino, E. M. 2001, ApJ, 560, 779

Chandler, C. J., \& Richer, J. S. 2001, ApJ, 555, 139

Davis, C. J., \& Smith, M. D. 1996, A\&A, 310, 961

Davis, C. J., Smith, M. D., \& Moriarty-Schieven, G. H. 1998, MNRAS, 299, 825

de Gouveia dal Pino, E. M., \& Benz, W. 1993, ApJ, 410, 686

Devine, D., Bally, J., Reipurth, B., \& Heathcote, S. 1997, AJ, 114, 2095

Downes, T. P., \& Cabrit, S. 2003, A\&A, 403, 135

Downes, T. P., \& Ray, T. P. 1999, A\&A, 345, 977

Draine, B. T., Roberge, W. G., \& Dalgarno, A. 1983, ApJ, 264, 485

Eislöffel, J. 2000, A\&A, 354, 236

Eislöffel, J., Smith, M. D., \& Davis, C. J. 2000, A\&A, 359, 1147

Frank, A., Lery, T., Gardiner, T. A., Jones, T. W., \& Ryu, D. 2000, ApJ, 540, 342

Frank, A., Ryu, D., Jones, T. W., \& Noriega-Crespo, A. 1998, ApJ, 494, L79

Froebrich, D., Smith, M. D., \& Hohapp, K. W., \& Eislöffel, J. 2003, A\&A, in press

Gómez de Castro, A. I., \& Robles, A. 1999, A\&A, 344, 632

Gardiner, T. A., Frank, A., Jones, T. W., \& Ryu, D. 2000, ApJ, 530, 834

Gueth, F., \& Guilloteau, S. 1999, A\&A, 343, 571

Gueth, F., Schilke, P., \& McCaughrean, M. J. 2001, A\&A, 375, 1018

Hartigan, P., Morse, J. A., Reipurth, B., Heathcote, S., \& Bally, J. 2001, ApJ, 559, L157 
Khanzadyan, T., Smith, M. D., Gredel, R., Stanke, T., \& Davis, C. J. 2002, A\&A, 383, 502

Lee, C., Mundy, L. G., Reipurth, B., Ostriker, E. C., \& Stone, J. M. 2000, ApJ, 542, 925

Lee, C., Mundy, L. G., Stone, J. M., \& Ostriker, E. C. 2002, ApJ, 576, 294

Lee, C., Stone, J. M., Ostriker, E. C., \& Mundy, L. G. 2001, ApJ, 557, 429

Lim, A. J., Raga, A. C., Rawlings, J. M. C., \& Williams, D. A. 2002, MNRAS, 335, 817

McKee, C. F., Storey, J. W. V., Watson, D. M., \& Green, S. 1982, ApJ, 259, 647

Nagar, N. M., Vogel, S. N., Stone, J. M., \& Ostriker, E. C. 1997, ApJ, 482, L195

Nisini, B., Caratti o Garatti, A., Giannini, T., \& Lorenzetti, D. 2002a, A\&A, 393, 1035

Nisini, B., Giannini, T., \& Lorenzetti, D. 2002b, ApJ, 574, 246

O’Sullivan, S., \& Ray, T. P. 2000, A\&A, 363, 355

Raga, A. C., Biro, S., Canto, J., \& Binette, L. 1991, Rev. Mex. Astron. Astrofis., 22, 243

Raga, A. C., Taylor, S. D., Cabrit, S., \& Biro, S. 1995, A\&A, 296, 833

Ray, T. P. 2000, Ap\&SS, 272, 115

Reipurth, B., \& Bally, J. 2001, ARA\&A, 39, 403

Reipurth, B., Bally, J., \& Devine, D. 1997, AJ, 114, 2708

Reipurth, B., Yu, K., Heathcote, S., Bally, J., \& Rodríguez, L. F. 2000, AJ, 120, 1449

Richer, J. S., Shepherd, D. S., Cabrit, S., Bachiller, R., \& Churchwell, E. 2000, Protostars and Planets IV, 867

Ridge, N. A., \& Moore, T. J. T. 2001, A\&A, 378, 495
Rosen, A., \& Smith, M. D. 2003, MNRAS, 343, 181

Russel, A. P. G., Bally, J., Padman, R., \& Hills, R. E. 1991, NASA STI/Recon Technical Report, 92, 22244

Salas, L., \& Cruz-González, I. 2002, ApJ, 572, 227

Saraceno, P., Andre, P., Ceccarelli, C., Griffin, M., \& Molinari, S. 1996, A\&A, 309, 827

Smith, M. D. 1994, MNRAS, 266, 238

Smith, M. D. 2000, Ir. Astron. J., 27, 25

Smith, M. D., Khanzadyan, T., \& Davis, C. J. 2003, MNRAS, 339, 524

Smith, M. D., \& Rosen, A. 2003, MNRAS, 339, 133

Smith, M. D., Suttner, G., \& Yorke, H. W. 1997, A\&A, 323, 223

Stanke, T. 2000, Ph.D. Thesis

Stanke, T., McCaughrean, M. J., \& Zinnecker, H. 2002, A\&A, 392, 239

Stone, J. M., \& Norman, M. L. 1993a, ApJ, 413, 198

Stone, J. M., \& Norman, M. L. 1993b, ApJ, 413, 210

Sutherland, R. S., Bicknell, G. V., \& Dopita, M. A. 2003, ApJ, 591, 238

Suttner, G., Smith, M. D., Yorke, H. W., \& Zinnecker, H. 1997a, A\&A, 318,595

Suttner, G., Smith, M. D., Yorke, H. W., \& Zinnecker, H. 1997b, in IAU Symp. 182

Völker, R., Smith, M. D., Suttner, G., \& Yorke, H. W. 1999, A\&A, 343, 953

Vannier, L., Lemaire, J. L., Field, D., et al. 2001, A\&A, 366, 651

Yu, K., Billawala, Y., Smith, M. D., Bally, J., \& Butner, H. M. 2000 , AJ, 120, 1974 